\title{
Genderspezifische Aspekte in der Sekundärprävention der koronaren Herzerkrankung
}

\author{
Oskar Mittag
}

Online publiziert: 1. März 2013

(C) Springer-Verlag Berlin Heidelberg 2013

\begin{abstract}
Zusammenfassung Der Faktor „Gender“ beinhaltet mindestens drei unterschiedliche Aspekte: biologische, psychosoziale (Lebenslagen) und Kohorteneffekte. Frauen und Männer unterscheiden sich hinsichtlich aller drei Bereiche. In dem Beitrag werden aktuelle Ergebnisse zu genderspezifischen Aspekten (z. B. Barrieren für körperliches Training, Belastungen und Krankheitsverarbeitung, Rehaziele und Präferenzen) der Sekundärprävention und Rehabilitation der koronaren Herzkrankheit in knapper Form dargestellt. Eine besondere Frage betrifft die Effekte von Programmen zur Sekundärprävention speziell für Frauen. Die Ergebnisse werden in den Kontext allgemeiner Probleme der Forschung zu Genderfragen gestellt.
\end{abstract}

Schlüsselwörter Gender · Koronare Herzkrankheit · Sekundärprävention

\section{Gender specific aspects of secondary prevention in coronary heart disease}

\begin{abstract}
Gender implies at least three different aspects: biological, psychosocial (life circumstances), and cohort effects. Women and men differ with respect to these areas. Current results as to gender specific aspects (e.g. barriers for physical exercise, stress and coping, rehabilitation goals and preferences) of secondary prevention and rehabilitation of patients with coronary heart disease are outlined. A specific question concerns the effects of secondary prevention programs tailored to meet women's needs.
\end{abstract}

Prof. Dr. O. Mittag $(\bowtie)$

Abteilung Qualitätsmanagement und Sozialmedizin (AQMS),

Universitätsklinikum Freiburg, Engelbergerstraße 21,

79106 Freiburg, Deutschland

E-Mail: oskar.mittag@uniklinik-freiburg.de
Results are discussed in the context of general problems of gender research.

Keywords Gender · Coronary heart disease · Secondary prevention

\section{Hintergrund}

Es gibt eine ganze Reihe von Mythen zu Geschlechtsunterschieden (nicht nur hinsichtlich der koronaren Herzerkrankung), die sich teilweise auch in der wissenschaftlichen Literatur hartnäckig halten. Dazu gehören im kardiologischen Bereich beispielsweise:

- Bei der koronaren Herzkrankheit und dem Herzinfarkt handelt es sich vorwiegend um eine Erkrankung von Männern. Tatsächlich sterben etwa 15\% der Frauen wie der Männer in Deutschland an einer ischämischen Herzerkrankung, wobei allerdings die Alterskurve bei den Frauen um 10 Jahre nach oben verschoben ist, während die Männer bei den KHK-Todesfällen unter 65 Jahren dominieren [1].

- Die Initialsymptomatik des akuten Koronarsyndroms gestaltet sich bei Frauen ,, atypisch “. Tatsächlich zeigen aktuelle Studien, dass sich Frauen und Männer kaum hinsichtlich der typischen Leitsymptomatik (Angina pectoris) unterscheiden, die Frauen aber darüber hinaus häufig noch weitere Symptome wie Übelkeit oder Rückenschmerzen schildern (z. B. [2]).

- Koronarkranke Frauen werden bei der medizinischen Behandlung benachteiligt (Koronarinterventionen, Medikation) und werden auch seltener als Männer in die kardiologische Rehabilitation überwiesen. Ersteres relativiert sich deutlich, wenn man das Alter berücksichtigt (vgl. [3]), und letzteres gilt zwar für viele Länder, in 
Deutschland dagegen stellt das Geschlecht zumindest in der Anschlussrehabilitation kein versorgungsrelevantes Merkmal dar (z. B. [4]).

Diese Beispiele zeigen, dass scheinbar genderspezifische Effekte oft mit dem Alter, der Komorbidität, dem Umgang mit Gesundheit und Krankheit sowie mit Systembedingungen (z. B. gesetzlichen Regelungen) zusammenhängen. Koronarkranke Frauen sind meist älter. Ihre Lebenslagen unterscheiden sich von denen der Männer (Erwerbstätigkeit, Bildung und Einkommen, Rolle und Verantwortlichkeiten in der Familie, Alleinleben). Sie haben mehr (und andere) Begleiterkrankungen und ihre körperliche Fitness ist geringer als die der Männer (vgl. [5]). Außerdem sind gerade jüngere Frauen, die psychisch besonders belastet sind (vgl. [6]), in der kardiologischen Rehabilitation zumeist in der Minderzahl. Bei der Untersuchung von Genderfragen und auch bei der Gestaltung der Sekundärprävention müssen diese Faktoren berücksichtigt werden.

\section{Sekundärprävention (Rehabilitation) bei Frauen und Männern}

\section{Körperliche Aktivität: Effekte und Barrieren}

Frauen profitieren hinsichtlich der Prognose der koronaren Herzerkrankung in gleichem Maße wie Männer von einem individuell dosierten Ausdauertraining (z. B. [7, 8]). Darüber hinaus reduziert Kraftausdauertraining insbesondere bei älteren Frauen den Verlust an Muskelmasse und Knochendichte [9]. Daraus resultiert die Empfehlung, dass Frauen wie Männer mehrmals in der Woche ein angepasstes regelmäßiges aerobes Ausdauertraining durchführen sollen, das durch ein leichtes dynamisches Krafttraining ergänzt wird [10].

Die körperliche Leistungsfähigkeit nach koronaren Ereignissen liegt bei Frauen aber deutlich unter derjenigen der Männer. In einer großen amerikanischen Untersuchung lag die Funktionskapazität von Frauen zu Beginn der kardiologischen Rehabilitation mit einer mittleren $\mathrm{VO}_{2}$ peak von etwa $15 \mathrm{ml} / \mathrm{kg} / \mathrm{min}$ um $5 \mathrm{ml} / \mathrm{kg} / \mathrm{min}$ niedriger als die von Männern [7]. Der Grund dürfte in der wesentlich geringeren sportlichen Aktivität von (älteren) Frauen liegen (vgl. [11]). Neben der geringeren Erfahrung mit sportlichen Aktivitäten bestehen aber noch weitere Barrieren für eine ausreichende körperliche Aktivität. Dazu zählen konkurrierende (familiäre) Verpflichtungen, Angst vor körperlicher Überlastung und Schmerzen, komorbide Erkrankungen (auch klimakterische Begleiterscheinungen) und eine eventuelle Inkontinenzproblematik [12]. Dies alles führt dazu, dass koronarkranke Frauen deutlich schwerer als Männer zu ausreichender körperlicher Aktivität zu motivieren sind.
Rehaziele und Versorgungsrealität

Die unterschiedlichen Lebenslagen von Frauen und Männern haben auch Konsequenzen für die (subjektiven) Ziele der Sekundärprävention. Frauen erwarten von der Rehabilitation Entlastung vom Alltag, Erhalt der Unabhängigkeit (Haushaltsführung), Gewichtsreduktion und emotionale Unterstützung. Bei Männern stehen dagegen körperliche Fitness, die Klärung beruflicher Probleme (Frühberentung) sowie die Erhöhung der Lebenserwartung im Vordergrund [5]. Das entspricht den unterschiedlichen Lebenslagen und Belastungen der beiden Geschlechter sowie dem verschiedenen Umgang mit Gesundheit und Krankheit.

Betrachtet man dagegen die Praxis der kardiologischen Rehabilitation, so wird deutlich, dass sich das Behandlungsangebot für Frauen und Männer nur geringfügig unterscheidet und deren unterschiedlichen Zielsetzungen nur unzureichend abbildet [13]. Hier stellt sich die Frage, ob Versorgungsgleichheit auch Bedarfsgerechtigkeit bedeutet. Kardiologische Rehabilitationsprogramme wurden ursprünglich mit Blick auf berufstätige Männer im mittleren Lebensalter entwickelt. Ob dieses Modell auch den Bedürfnissen und Präferenzen von koronarkranken Frauen entspricht, ist fraglich. In Tab. 1 werden typische Belastungen und Bewältigungsstrategien bei (koronarkranken) Frauen und Männern aufgelistet und Möglichkeiten einer genderspezifischen Gestaltung von Maßnahmen zur Sekundärprävention skizziert.

\section{Sekundärprävention speziell für Frauen}

Es gibt inzwischen, insbesondere aus dem skandinavischen Raum, eine Reihe von randomisierten, kontrollierten Studien, in denen Rehaprogramme evaluiert wurden, die speziell auf die Bedürfnislagen von Frauen zugeschnitten waren. Dahinter steht die Überlegung, dass koronarkranke Frauen(-herzen) einer besonderen Behandlung bedürfen - „women's hearts need special treatment“ [14, S. 228]. Kernpunkt dieser Programme war zumeist ein Stressbewältigungsprogramm, dessen Inhalte auf die spezifischen Lebenslagen und Bedürfnisse von Frauen zugeschnitten waren (z. B. Umgang mit multiplen Rollen, Bewältigung von Alltagsproblemen).

In den Studien zeigten sich positive Effekte hinsichtlich der (subjektiven) körperlichen Gesundheit, der Depressivität und der vitalen Erschöpfung [15, 16], nicht aber bezüglich physiologischer Parameter für das koronare Risiko [17]. In der einzigen deutschen Studie [18] führte die Teilnahme an einer reinen Frauengruppe zu höherer Zufriedenheit und langfristig zu mehr körperlicher Aktivität. Effekte einer frauenspezifischen psychosozialen Intervention hinsichtlich sog. „harter“ Endpunkte wurden im Rahmen der SWITCHD-Studie [19] gefunden. Die Intervention bestand 
Tab. 1 Typische Belastungen, Krankheitsbewältigung und Rehaziele bei Frauen und Männern sowie Möglichkeiten einer genderspezifischen Gestaltung von medizinischer Rehabilitation. (Mod. nach [5])

\begin{tabular}{|c|c|c|}
\hline Bereich & Frauen & Männer \\
\hline Belastungen und Probleme & $\begin{array}{l}\text { Umgang mit Mehrfachbelastungen } \\
\text { Selbstorganisation/Zeitmanagement } \\
\text { Stress in der Familie (,,marital stress“) } \\
\text { Barrieren für Sport und Bewegung }\end{array}$ & $\begin{array}{l}\text { Berufliche Problemlagen } \\
\text { Gratifikationskrisen } \\
\text { Gelingen von Beziehungen (spez. im Alter) }\end{array}$ \\
\hline Krankheitsbewältigung & $\begin{array}{l}\text { Krankheitsbedingte Sorgen } \\
\text { Depressivität (insbes. bei jüngeren) } \\
\text { Nutzung sozialer Ressourcen } \\
\text { Krankheit schicksalhaft }\end{array}$ & $\begin{array}{l}\text { Selbstwirksamkeitserwartungen/,Kampfgeist“ } \\
\text { Befolgen der ärztlichen Behandlung }\end{array}$ \\
\hline Subjektive Rehaziele & $\begin{array}{l}\text { Entlastung vom Alltag/Erholung } \\
\text { Erhalt der Unabhängigkeit (Haushalt) } \\
\text { Gewichtsreduktion }\end{array}$ & $\begin{array}{l}\text { Schnelle Genesung } \\
\text { Fitness/Muskelaufbau } \\
\text { Klärung beruflicher Probleme/Rente } \\
\text { Erhöhung der Lebenserwartung }\end{array}$ \\
\hline Interventionsstrategien & $\begin{array}{l}\text { Emotionale Unterstützung } \\
\text { Bewältigung spezifischer Stressbelastungen }\end{array}$ & $\begin{array}{l}\text { Instrumentelle Unterstützung } \\
\text { Beratung zur Lebensstiländerung }\end{array}$ \\
\hline $\begin{array}{l}\text { Differenzierung der } \\
\text { Angebote }\end{array}$ & $\begin{array}{l}\text { Nach Alter } \\
\text { Nach Lebenslagen }\end{array}$ & - \\
\hline Organisation/Strukturen & $\begin{array}{l}\text { „Reine“ Frauengruppen } \\
\text { Eher stationär und wohnortfern } \\
\text { Betreuung von pflegebedürftigen Angehörigen? }\end{array}$ & $\begin{array}{l}\text { „Anonyme“ Angebote? } \\
\text { Web-basierte Interventionen? }\end{array}$ \\
\hline
\end{tabular}

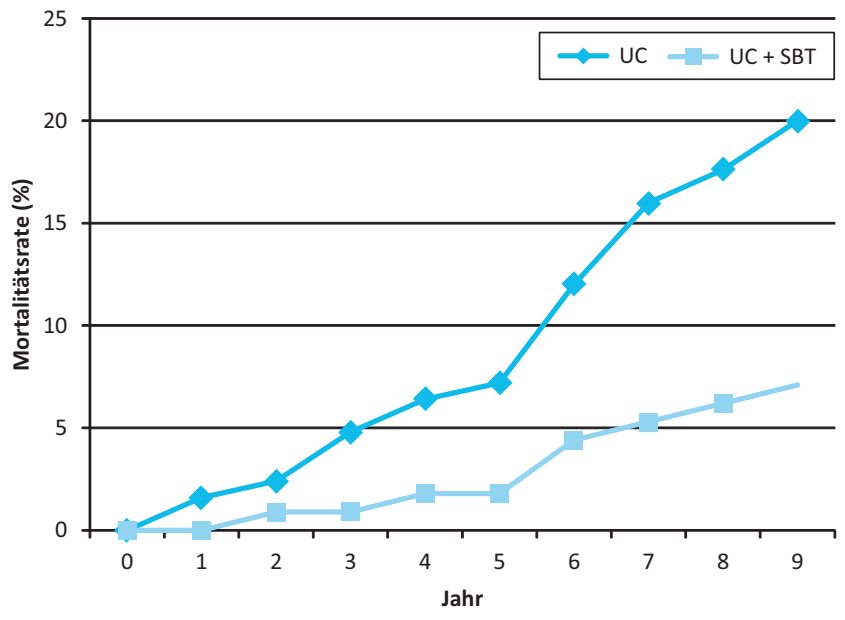

Abb. 1 Ergebnisse aus der SWITCHD-Studie: Gesamtmortalität über 9 Jahre; $U C$ usual care, $U C+S B T$ zusätzliches Stressbewältigungstraining. (Daten aus [19])

in einem kognitiv-behavioralen Stressbewältigungsprogramm mit 20 Kleingruppensitzungen über einen Zeitraum von 12 Monaten. Die Kontrollgruppe erhielt die übliche kardiologische Behandlung. Im (mittleren) Katamnesezeitraum von 7 Jahren war die Gesamtmortalität in der Interventionsgruppe um 17 Prozentpunkte geringer als in der Kontrollgruppe (vgl. Abb. 1).

Brauchen Frauen und Männer andere Kommunikationsstrategien?

Ende der 1990er Jahre wurden Ergebnisse aus dem „Montreal Heart Attack Trial“ (M-HART) bekannt, die andeu- teten, dass die Intervention (Telefonate und Hausbesuche durch speziell geschultes Pflegepersonal) bei den Frauen in der Stichprobe möglicherweise zu einer erhöhten Mortalität geführt hatte [20]. Eine weitere große randomisierte Studie (ENRICHD) ergab, dass v. a. (weiße) Männer hinsichtlich der kardialen Morbidität und Mortalität von der Intervention profitiert hatten, nicht jedoch die Frauen in der Stichprobe [21]. Ein ähnliches Ergebnis zeigte sich in einer Rehanachsorgestudie hinsichtlich des koronaren Risikos [22]. Eine aktuelle Studie zur Behandlung komorbider Depression bei Patienten nach ACVB-Operation fand ebenfalls, dass die Männer, nicht aber die Frauen hinsichtlich des psychischen Befindens profitierten [23].

Diese Befunde irritieren in hohem Maße. Wie kommt es, dass (koronarkranke) Frauen und Männer anscheinend ganz unterschiedlich von psychosozialen Angeboten profitieren? Nachträgliche Analysen der Daten aus M-HART [24] legen nahe, dass Frauen und Männer auf bestimmte Interventionen je nach Thema unterschiedlich reagieren; Männer profitierten demzufolge eher von Informationen und Ratschlägen hinsichtlich ihres Gesundheitsverhaltens, während Frauen eher auf Zuhören und unterstützende Interventionen hinsichtlich ihrer Alltagsbelastungen ansprachen. Hier handelt es sich aber um singuläre Ergebnisse. Weitere Studien zu den Kommunikationspräferenzen von Frauen und Männern wären unbedingt wünschenswert.

\section{Fazit für die Praxis}

Bei der Berücksichtigung genderspezifischer Aspekte in der Sekundärprävention (Rehabilitation) der koronaren Herz- 
krankheit geht es vor allem darum, den besonderen Lebenslagen und Bedürfnisse koronarkranker Frauen Rechnung zu tragen. Das gilt z. B. für die Inhalte und das Setting der Rehabilitation (einschließlich der Nachsorge) sowie in besonderem Maße für die Sensibilität hinsichtlich der Probleme (älterer) Frauen in der Bewegungstherapie. Jüngere Frauen kommen zahlenmäßig selten in die kardiologische Rehabilitation. Gerade diese Gruppe ist aber psychisch besonders belastet. Auch das sollte bei der Rehabilitation berücksichtigt werden, indem z. B. jüngere Frauen schwerpunktmäßig in Rehaeinrichtungen behandelt werden, die spezielle Programme für diese Zielgruppe anbieten.

Insgesamt haben wir erstaunlich wenig gesichertes Wissen über genderspezifische Aspekte. Das gilt nicht nur für die Sekundärprävention der koronaren Herzkrankheit. Eine bewusste und kontinuierliche Berücksichtigung von Genderaspekten in der Forschung ist daher erforderlich. Der Faktor Gender beinhaltet dabei mindestens drei unterschiedliche Aspekte: Geschlecht stellt zunächst eine biologische Variable dar, es ist aber auch mit unterschiedlichen Lebenslagen verbunden und bildet auch Kohorteneffekte ab, die insbesondere bei Frauen ja nach Alter bzw. Generation mit deutlich unterschiedlichen Rollenbildern (z. B. bezüglich sportlicher Aktivitäten) verbunden sind. Werden diese Faktoren nicht angemessen berücksichtigt, besteht immer die Gefahr, beispielsweise die Effekte von Alter oder sozialen Lebenslagen fälschlicherweise als Gendereffekte zu interpretieren. Diese Komplexität macht die Beschäftigung mit Genderfragen zu einem schwierigen, gerade deswegen aber auch ungemein reizvollen Forschungsfeld.

Acknowledgments This article is part of a supplement sponsored by Lilly Deutschland GmbH and Daiichi Sankyo Deutschland GmbH.

Interessenkonflikt Der korrespondierende Autor gibt für sich und seine Koautoren an, dass kein Interessenkonflikt besteht.

\section{Literatur}

1. Statistisches Bundesamt (2011) Statistisches Jahrbuch. Statistisches Bundesamt, Wiesbaden

2. Kirchberger I, Heier M, Kuch B, Wende R, Meisinger C (2011) Sex differences in patient-reported symptoms associated with myocardial infarction (from the population-based MONICA/KORA Myocardial Infarction Registry). Am J Cardiol 107:1585-1589

3. Williams RI, Fraser AG, West R (2004) Gender differences in management after acute myocardial infarction: not ,sexism' but a reflection of age at presentation. J Public Health 26:259-263

4. Grande G, Leppin A, Romppel M, Altenhöner T et al (2002) Frauen und Männer nach Herzinfarkt: Gibt es in Deutschland geschlechtsspezifische Unterschiede in der Inanspruchnahme rehabilitativer Leistungen? Rehabilitation 41:320-328

5. Mittag O, Grande G (2008) PatientInnenorientierung in der Rehabilitation: Die Genderperspektive. Rehabilitation 47:98-108
6. Beckie TM, Fletcher GF, Beckstead JW, Schocken DD et al (2008) Adverse baseline physiological and psychosocial profiles of women enrolled in a cardiac rehabilitation clinical trial. J Cardiopulm Rehabil Prev 28:52-60

7. Ades PA, Savage PD, Brawner CA, Lyon CE et al (2006) Aerobic capacity in patients entering cardiac rehabilitation. Circulation 113:2706-2712

8. Kavanagh T, Mertens DJ, Hamm LF, Beyene J et al (2003) Peak oxygen intake and cardiac mortality in women referred for cardiac rehabilitation. J Am Coll Cardiol 42:2139-2143

9. Shea B, Bonaiuti D, Iovine R, Negrini S et al (2004) Cochrane Review on exercise for preventing and treating osteoporosis in postmenopausal women. Eura Medicophys 40:199-209

10. Bjarnason-Wehrens B, Schulz O, Gielen S, Halle M et al (2009) Leitlinie körperliche Aktivität zur Sekundärprävention und Therapie kardiovaskulärer Erkrankungen. Clin Res Cardiol Suppl 4:1-44

11. Mensink G (2003) Bundes-Gesundheitssurvey: Körperliche Aktivität. Robert Koch-Institut, Berlin

12. Bjarnason-Wehrens B, Grande G, Loewel H, Voeller H, Mittag O (2007) Gender specific issues in cardiac rehabilitation: Do women with ischemic heart disease need specially tailored programs? Eur J Cardiovasc Prev Rehabil 14:163-171

13. Bitzer EM, Klosterhuis H, Dörning H, Rose S (2003) Entwicklung einer evidenzbasierten Leitlinie zur kardiologischen Rehabilitation: Phase 2: Vergleichende Analyse des Ist-Zustandes der in der kardiologischen Rehabilitation erbrachten Leistungen auf der Basis der KTL-Statistik. Rehabilitation 42:83-93

14. Burell G, Granlund B (2002) Women's hearts need special treatment. Int J Behav Med 9:228-242

15. Beckie TM, Beckstead JW, Schocken DD, Evans ME, Fletcher GF (2011) The effects of a tailored cardiac rehabilitation program on depressive symptoms in women: a randomized clinical trial. Int J Nurs Stud 48:3-12

16. Claesson M, Birganda LS, Lindahl B, Nasic S, Aström M, Asplund K, Burell G (2005) Women's hearts - stress management for women with ischemic heart disease. J Cardiopulm Rehabil 25:93-102

17. Claesson M, Birgander LS, Jansson JH, Lindahl B et al (2006) Cognitive-behavioural stress management does not improve biological cardiovascular risk indicators in women with ischaemic heart disease: a randomized-controlled trial. J Intern Med 260:320-331

18. Härtel U, Klein G (2007) Körperliche Aktivität von Frauen im Jahr nach akuter koronarer Herzkrankheit: Ergebnisse einer Frauen-Interventionsstudie in der kardiologischen Rehabilitation. In: Deutsche Rentenversicherung Bund (Hrsg) 16. Rehabilitationswissenschaftliches Kolloquium DRV-Schriften Bd 72. DRV Eigenpublikation, S 223-224

19. Orth-Gomér K, Schneiderman N, Wang HX, Walldin C, Blom M, Jernberg T (2009) Stress reduction prolongs life in women with coronary heart disease. The Stockholm Women's Intervention Trial for Coronary Heart Disease (SWITCHD). Circ Cardiovasc Qual Outcomes 2:25-32

20. Frasure-Smith N, Lespérance F, Prince RH, Verrier P et al (1997) Randomised trial of home-based psychological nursing intervention for patients recovering from myocardial infarction. Lancet 350:473-479

21. Schneiderman N, Saab PG, Catellier DJ, Powell LH et al (for the ENRICHD Investigators) (2004) Psychosocial treatment with sex by ethniticy subgroups in the enhancing recovery in coronary heart disease clinical trial. Psychosom Med 66:475-483

22. Mittag O, China C, Hoberg E, Juers E et al (2006) Outcomes of a telephone counseling Intervention following cardiac rehabilitation (Luebeck follow-up trial): overall and gender specific results. Int J Rehabil Res 29:295-302 
23. Rollman BL, Herbeck Belnap B (2011) The bypassing the blues trial: collaborative care for post-CABG depression and implications for future research. Cleve Clin J Med 78:4-12
24. Cossette S, Frasure-Smith N, Lespérance F (2002) Nursing approaches to reducing psychological distress in men and women recovering from myocardial infarction. Int J Nurs Stud $39: 479-494$ 